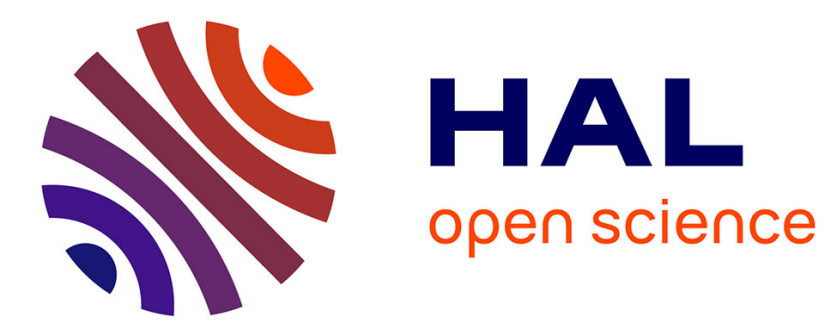

\title{
Complete Electroelastic Set of Co doped Barium Titanate for Transducer Applications
}

Rémy Ul, Rémi Rouffaud, Louis-Pascal Tran-Huu-Hue, Franck Levassort, Maï Pham-Thi, Claire Bantignies

\section{- To cite this version:}

Rémy Ul, Rémi Rouffaud, Louis-Pascal Tran-Huu-Hue, Franck Levassort, Maï Pham-Thi, et al.. Complete Electroelastic Set of Co doped Barium Titanate for Transducer Applications. 2017 IEEE International Ultrasonics Symposium (IUS), Sep 2017, Washington, DC, United States. 10.1109/ULTSYM.2017.8091772 . hal-01705122

\section{HAL Id: hal-01705122 \\ https://hal.science/hal-01705122}

Submitted on 9 Feb 2018

HAL is a multi-disciplinary open access archive for the deposit and dissemination of scientific research documents, whether they are published or not. The documents may come from teaching and research institutions in France or abroad, or from public or private research centers.
L'archive ouverte pluridisciplinaire $\mathbf{H A L}$, est destinée au dépôt et à la diffusion de documents scientifiques de niveau recherche, publiés ou non, émanant des établissements d'enseignement et de recherche français ou étrangers, des laboratoires publics ou privés. 


\section{Complete Electroelastic Set of co doped Barium Titanate for Transducer Applications}

\author{
Rémy U1 ${ }^{1,2}$, Rémi Rouffaud ${ }^{1}$ \\ Louis-Pascal Tran-Huu-Hue ${ }^{1}$, Franck Levassort ${ }^{1}$ \\ TOURS University, GREMAN UMR 7347, CNRS, \\ INSA-CVL, Blois, France \\ remy.ul@insa-cvl.fr
}

\author{
Mai Pham-Thi ${ }^{2}$ \\ ${ }^{2}$ Thales Research\&Technology-France \\ Palaiseau, France \\ Claire Bantignies ${ }^{3}$ \\ ${ }^{3}$ Advanced Research dept., VERMON SA \\ Tours, France
}

\begin{abstract}
BaTiO}_{3}$ ceramic doped with cobalt and calcium (BTCaCo), processed by a conventional technique with a reduced sintering temperature, is characterized. Particular attention is paid to the delivery of complete and accurate set of elastic, dielectric, and piezoelectric constants. These databases are relatively rare in the literature but are essential for numerical simulations of new devices, integrating these materials. The processed piezoelectric material exhibits an electromechanical thickness coupling factor $\left(k_{t}\right)$ of $45 \%$ and a relative dielectric constant of 1180, at constant strain. A 1-3 piezo-composite is successfully fabricated to improve the performance $\left(k_{t}=47 \%\right)$. Finally, BTCaCo disc is used to fabricate a transducer with a center frequency at $4.3 \mathrm{MHz}$, showing competitive performance compared to a standard PZT-based transducer.
\end{abstract}

Keywords-barium titanate; piezoelectricity; functional characterization; piezocomposite.

\section{INTRODUCTION}

Barium titanate $\left(\mathrm{BaTiO}_{3}\right)$ is a well-known ferroelectric material that was intensively studied in the early 50's [1]. Indeed, $\mathrm{BaTiO}_{3}$ has been rapidly substituted by lead zirconate titanate (PZT), which exhibits higher piezoelectric properties. However, PZT material contains lead and their increasing success is associated to health and environmental problems. Therefore, efforts have been devoted to the development of competitive lead-free counterparts and consequently, $\mathrm{BaTiO}_{3}$ compositions have comeback on center stage as promising candidates. Various papers have been reported on $\mathrm{BaTiO}_{3}$ based materials, processed by employing conventional solidstate techniques, with improved piezoelectric properties [2]. Here, barium titanate co-doped with cobalt/calcium (BTCaCo) is prepared by conventional solid-state reaction method. Three samples with different geometries including pellets with a thickness of $0.6 \mathrm{~mm}$ and a diameter of $13.5 \mathrm{~mm}$ are fabricated. In order to obtain the complete set of material constants, resonance-antiresonance method is first used. In this case, measured and calculated values are mixed. This can lead to violations in the interrelations [3] between particular groups of electroelastic moduli and non-consistent database is provided. In this study, a method based on a genetic algorithm is used to optimize the consistency of the full set of material constants

This work was supported by the French Research Agency (HEcATE, ANR 14-CE07-0028-01) through the minimization of a defined criterion. This method has already been applied to $\mathrm{KN}$ single crystal characterization [4]. With a BTCaCo disc, a single element tranducer is fabricated and the measured performances are compared with the theoretical behavior to evaluate the efficiency of this leadfree composition. Then, 1-3 piezocomposite is fabricated to evaluate the possible improved properties in this configuration for transducer applications. The following section describes the fabrication of ceramic and composite samples. Section III is devoted to the database of BTCaCo material. Finally, the last part (section IV), gives the electromechanical performance of the 1-3 piezocomposite and acoustic properties of the transducer.

\section{FABriCATION AND ELECTRICAL MEASUREMENTS}

\section{A. Ceramic samples fabrication}

Co-doped barium titanate is fabricated using oxide and carbonate powder as raw materials: $\mathrm{BaCO}_{3}, \mathrm{TiO}_{2}, \mathrm{Co}_{3} \mathrm{O}_{4}$, $\mathrm{CaCO}_{3}$ and $\mathrm{Li}_{2} \mathrm{O}$. The calcium dopant is introduced in substitution to the barium and the cobalt in substitution to the titanium. According to the chemical formula $\left(\mathrm{Ba}_{0.99} \mathrm{Ca}_{0.01}\right)\left(\mathrm{Ti}_{0.9925} \mathrm{Co}_{0.0075}\right) \mathrm{O}_{3-\delta}$ the powders are weighed, mixed, and then ball-milled by attrition in water for $1 \mathrm{~h}$. The mixture is then dried calcined at $1100{ }^{\circ} \mathrm{C}$ for $2 \mathrm{~h}$. Thereafter, calcined powders are ball-milled by attrition for $0.5 \mathrm{~h}$, dried, and then mixed with $1 \mathrm{~mol} \%$ of $\mathrm{Li}_{2} \mathrm{O}$ powder and with polyvinyl butyral and dibutyl phthalate used as a binder and a plasticizer. The resulting powder is then pressed by uniaxial pressing into a cylinder of $16.3 \mathrm{~mm}$ in diameter and $10-10.5$ $\mathrm{mm}$ in thickness. After burning the binder and plasticizer at $600{ }^{\circ} \mathrm{C}$ for $0.5 \mathrm{~h}$, the pressed cylinder is sintered at $1100{ }^{\circ} \mathrm{C}$ for $4 \mathrm{~h}$ in air. After sintering, the ceramic is cut into either several $0.6 \mathrm{~mm}$ thickness pellets or bars of $9 \mathrm{~mm}^{2}$ in surface and $9 \mathrm{~mm}$ in height or platelets of $3 \mathrm{~mm}$ width $12 \mathrm{~mm}$ length and $0.55 \mathrm{~mm}$ thick. The geometries of the samples are designed to ensure different modes of vibration of the ceramic, such as thickness or planar (Fig. 1) and to give insight to the different constants associated with that modes.

The samples are coated with silver paint and fired at 500 ${ }^{\circ} \mathrm{C}$ for $1 \mathrm{~h}$. The coated samples are then poled in a silicone oil 
bath by Field Cooling, consisting with the applying of a DC electric field of $600 \mathrm{~V} / \mathrm{mm}$ during the cooling process of the samples from $140-150{ }^{\circ} \mathrm{C}$ to $40-50{ }^{\circ} \mathrm{C}$.
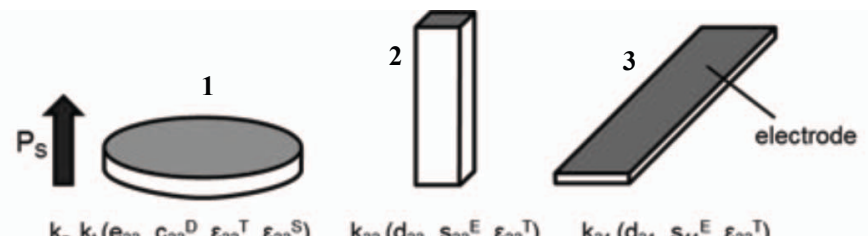

Fig. 1. Schematic representations of the different geometries needed to access to a complete electroelastic moduli.

\section{B. 1-3 Piezocomposite fabrication}

Dice and fill method [5] is used for the fabrication of 1-3 piezocomposite from a $\mathrm{BTCaCo}$ disc. Dicing conditions are tuned for this material which has slightly higher elastic parameters than conventional lead-based ceramics. A ceramic volume fraction of $50 \%$ is chosen with a kerf around $30 \mu \mathrm{m}$. Samples are lapped to reduce the thickness to reach $400 \mu \mathrm{m}$. Finally, gold electrodes are sputtered on both faces (Fig. 2).

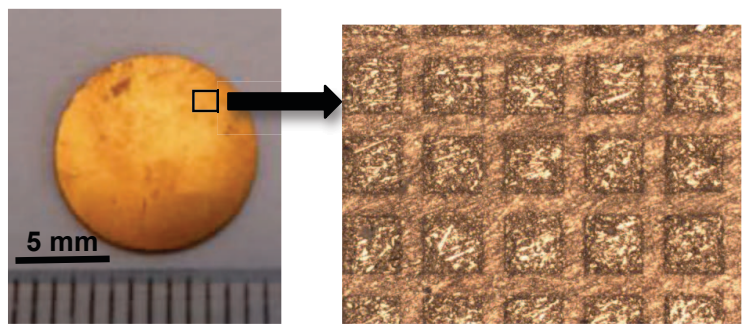

Fig. 2. Optical photographs of (a) fabricated 1-3 piezocomposite disc (top view) and (b) zoom of the structure (squares : ceramic rods surrounded by epoxy resin).

\section{Electrical measurements}

Measurements of the complex electrical impedance around the fundamental resonance of the favor mode (i.e. IEEE standard [6] are performed with an Agilent 4395A or 4294A impedance spectrum analyzer (Agilent Technologies Inc., Palo Alto, CA) and an impedance test kit. For the three BTCaCo samples, resonance and antiresonant frequencies are measured from maximum values of electrical admittance and impedance, respectively. According to relations given in [3], thickness $\left(\mathrm{k}_{\mathrm{t}}\right)$ and planar $\left(\mathrm{k}_{\mathrm{p}}\right)$ coupling factors are deduced with sample 1 , $\mathrm{k}_{33}$ with sample 2 and $\mathrm{k}_{31}$ with sample 3 (Fig. 1 ).

For the characterization of the 1-3 piezocomposite, the KLM equivalent electrical circuit [7] is used to compute the theoretical behavior of the electrical impedance and a fitting process to deduce the thickness-mode parameters of the sample (Fig.2). In this case, five parameters are evaluated: the loss factors (mechanical, $\delta \mathrm{m}$, and electrical, $\delta \mathrm{e}$ ), the effective thickness coupling factor kt, the longitudinal wave velocity $\mathrm{c}_{\mathrm{L}}$ and the dielectric constant at constant strain.
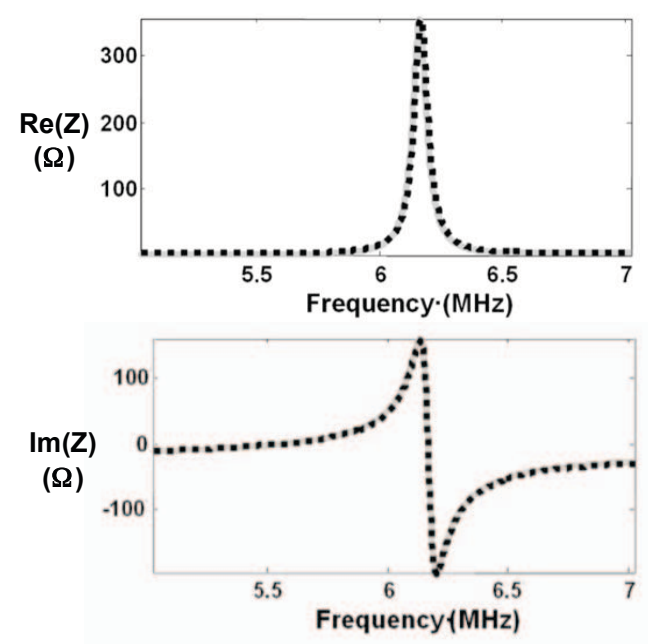

Fig. 3. Complex electrical impedance $(Z)$ of the BTCaCo based 1-3 piezocomposite (gray solid lines : theoretical curves with KLM scheme, dashed black lines : experimental curves).

\section{COMPLETE SET OF Co DOPED BT}

For modeling ultrasonic transducers with numerical methods such as Finite Element Method, all components from elastic, dielectric and piezoelectric tensors are required. Depending on the calculation performed, only one subset of tensors of the four possible ones is chosen (for example, $s^{\mathrm{E}}, \mathrm{d}$ and $\varepsilon^{\mathrm{T}}$ ). Regardless of this choice, the calculation has to give a unique and similar result. If the result depends on the choice of the subset, the complete set is inconsistent [8, 4]. The complete set provided in this paper avoids this important defect.

\section{A. Consistency and quantification criterion}

In order to provide a complete and consistent set, all of the 16 intercoefficient relations [3] that allow switching from a subset to another, must be respected. Because of the measured parameters, experimental inconsistencies can be introduced in the complete set due to the experimental method chosen. So, intercoefficient relations cannot be strictly respected but, in the method reported here, particular attention will be paid to reduce these errors.

Among the 40 constants that make a $6 \mathrm{~mm}$ structure database, 18 constants are measured or calculated in a first step (part II.C) and are indicated in Table I with stars. So, 22 values have to be determined to complete the material set. The genetic algorithm (GA) [9] is used to examine the wide search space created by these 22 parameters. In order to limit the search space without damaging the quality of the solution, upper and lower boundaries are defined for the investigated values between 0 and 2 times the reference values. In this work, reference values are taken from the Bechmann's results [10] about the complete electroelastic set of barium titanate ceramic because of the similar compositions. The GA attempts to reduce the fitness value $p$ as it is defined in [4]. Relative distances are calculated between both right- and left-hand sides of each intercoefficient relation. The value $p$ represents 
the maximum values of these relative distances and gives an indication of the consistency of the delivered full database.

\section{B. Determination of the electroelastic moduli}

The complete set of obtained material parameters is given in Table II (last page). As mentioned before, 18 parameters are measured and are marked with a star. The maximum $p$ value is found at $7.4 \%$ for the following intercoefficient relation (only for $\mathrm{k}=3$ and $\mathrm{q}=3$ ):

$$
g_{k q}=h_{k p} s_{p q}^{D}
$$

More precisely, the value of the left member $\left(g_{33}\right)$ of the relation (1) is equal to $0.0123 \mathrm{~V} \cdot \mathrm{mN}^{-1}$ while the right member has the value $0.0130 \mathrm{~V} \cdot \mathrm{mN}^{-1}$.

This $p$-value obtained is in agreement with those that can be found in the literature calculated from the available database. As example, one of the best complete electroelastic sets for a lead-free single crystal [11] exhibits a $p$-value of $8.5 \%$.

\section{1-3 PIEZOCOMPOSITE AND TRANSDUCERS}

Table II summarizes the electromechanical properties of 1-3 piezocomposite for the thickness mode. The resonant frequency in air is at $6.1 \mathrm{MHz}$ for a thickness around $400 \mu \mathrm{m}$. According to the design of the piezocomposite (pitch and kerf values), the thickness mode is not disturbed by the first lateral mode, as shown in Fig. 3. The corresponding coupling factor slightly increases compared to the ceramic (from $45 \%$ to $47 \%$ ) but do not reach the $\mathrm{k}_{33}$ value of the ceramic given in Table I.

TABLE I. FUNCTIONAL PROPERTIES OF BTCACO-BASED 1-3 PIEZOCOMPOSITE

\begin{tabular}{|c|c|c|c|c|c|c|}
\hline $\begin{array}{c}\boldsymbol{t} \\
(\mu \mathrm{m})\end{array}$ & $\begin{array}{c}\boldsymbol{A} \\
\left(\mathrm{mm}^{2}\right)\end{array}$ & $\boldsymbol{\varepsilon}_{33} \boldsymbol{s} / \boldsymbol{\varepsilon}_{\boldsymbol{0}}$ & $\begin{array}{c}\boldsymbol{k}_{\boldsymbol{t}} \\
(\%)\end{array}$ & $\begin{array}{c}\boldsymbol{\delta}_{\boldsymbol{m}} \\
(\%)\end{array}$ & $\begin{array}{c}\boldsymbol{\delta}_{e} \\
(\%)\end{array}$ & $\boldsymbol{Z}(M R a)$ \\
\hline 404 & 95 & 580 & 47 & 1.5 & 1 & 17.1 \\
\hline
\end{tabular}

${ }^{\mathrm{a}} \mathrm{t}$ : thickness; A: area of the sample; $\varepsilon_{33}{ }^{\mathrm{S}} / \varepsilon_{0}$ : relative dielectric constant at constant strain; $\mathrm{k}_{\mathrm{t}}$ : effective thickness coupling factor; $\delta_{\mathrm{m}}$ : mechanical losses; $\delta_{\mathrm{e}}$ : dielectric losses; $\mathrm{Z}$ : acoustical impedance.

With BTCaCo disc (thickness of $588 \mu \mathrm{m}$ and area of 140 $\mathrm{mm}^{2}$ ), a single-element transducer is fabricated. The backing is made with epoxy resin loaded with tungsten powder $(10 \%$ volume fraction) to deliver an acoustical impedance of 5.2 MRa. No matching layer is added. Two electrical contacts are made on each electrode with thin copper wires and conductive epoxy resin. The transducer is inserted into an insulating housing (Fig. 4). A coaxial cable with the characteristic impedance of $\mathrm{Z}_{\mathrm{c}}=50 \Omega$ and a length of around $40 \mathrm{~cm}$ is used for the electrical connection to the pulser/receiver (PR5900, panametrics). The transducer is placed in water in front of a metallic target to measure the electroacoustic response. It is recorded with an oscilloscope (Lecroy Waverunner 64XI).

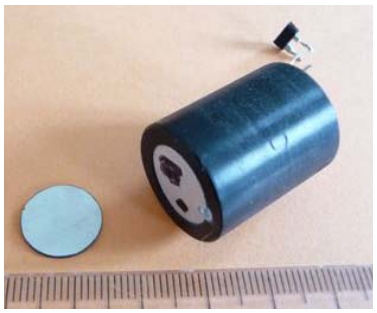

Fig. 4. Photography of the fabricated transducer including BTCaCo disc.

Fig. 5 shows this experimental response which has a center frequency of $4.8 \mathrm{MHz}$. According to the low backing acoustical impedance used with no matching layer, fractional bandwidth is logically limited $(20 \%$ at $-6 \mathrm{~dB}$ and $59 \%$ at -20 $\mathrm{dB})$. With the KLM scheme [6], this response is modeled with good accuracy (Fig. 5) and same sensitivity.

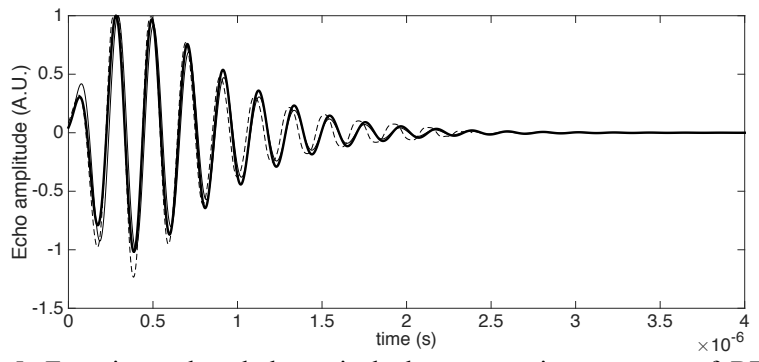

Fig. 5. Experimental and theoretical electroacoustic responses of BTCaCo based transducer (dashed black line: experimental; thick solid black line: theoretical) and Pz27 theoretical thin solid black line).

Finally, the BTCaCo disc is replaced by a standard PZT (Pz27, MEGGITT A/S [12]) with the same area. Only the thickness is adapted to keep the same center frequency. This last theoretical electroacoustic response is superimposed with the two previous ones (Fig. 5). Differences of sensitivity and bandwidth $(20.7 \%$ at $-6 \mathrm{~dB})$ are low.

\section{CONCLUSIONS}

Co doped barium titanate $(\mathrm{BTCaCo})$ was fabricated with an objective of a preindustrial process in particular with a low sintering temperature while keeping good electromechanical performance. Full consistent database (40 elastic, dielectric and piezoelectric parameters) of BTCaCo was obtained with, for example a thickness coupling factor $\left(\mathrm{k}_{\mathrm{t}}\right)$ of $45 \%$. Piezocomposites with a ceramic volume fraction of $50 \%$ were successfully fabricated, thanks to a dense microstructure and low grain size. The BTCaCo-based transducer, with a low acoustical impedance backing (5.2 MRa), possess a fractional bandwidth $(-6 \mathrm{~dB})$ at $20 \%$ and a resonant frequency at 4.8 $\mathrm{MHz}$. These results were in good agreement with simulated transducer behaviors using KLM scheme. They were compared with a transducer based on a standard PZT pellet to deliver similar sensitivities and bandwidth showing the efficiency of co-doped barium titanate. 


\begin{tabular}{|c|c|c|c|c|c|}
\hline \multicolumn{6}{|c|}{$(S, E)$ - type fundamental relations : $c_{i j}^{E}\left(10^{9} \mathrm{~Pa}\right), e_{i j}\left(\mathrm{Cm}^{-2}\right), \varepsilon_{i j}^{S} / \varepsilon_{0}$} \\
\hline$c_{11}^{E *}$ & $c_{12}^{E}$ & $c_{13}^{E}$ & $c_{33}^{E} *$ & $c_{44}^{E}$ & $c_{66}^{E}$ \\
\hline 150 & 66.94 & 54.26 & 138.9 & 40.84 & 41.53 \\
\hline$e_{31}$ & $e_{33} *$ & $e_{15}$ & & $\varepsilon_{11}^{S}$ & $\varepsilon_{33}^{S} *$ \\
\hline-8.90 & 19.31 & 13.14 & & 1215 & 1179 \\
\hline \multicolumn{6}{|c|}{$(S, D)$ - type fundamental relations : $c_{i j}^{D}\left(10^{9} \mathrm{~Pa}\right), h_{i j}\left(10^{9} \mathrm{Vm}^{-1}\right), \beta_{i j}^{S}\left(10^{9} \mathrm{mF}^{-1}\right)$} \\
\hline$c_{11}^{D}$ & $c_{12}^{D}$ & $c_{13}^{D}$ & $c_{33}^{D *}$ & $c_{44}^{D}$ & $c_{66}^{D}$ \\
\hline 157.59 & 74.58 & 38.89 & 174.70 & 56.42 & 41.50 \\
\hline$h_{31}$ & $h_{33}$ & $h_{15}$ & & $\beta_{11}^{S}$ & $\beta_{33}^{S}$ \\
\hline-0.85 & 1.73 & 1.21 & & 0.093 & 0.089 \\
\hline \multicolumn{6}{|c|}{$(T, E)$ - type fundamental relations : $s_{i j}^{E}\left(10^{-12} \mathrm{~m}^{2} \mathrm{~N}^{-1}\right), d_{i j}\left(10^{-12} \mathrm{CN}^{-1}\right), \varepsilon_{i j}^{T} / \varepsilon_{0}$} \\
\hline$s_{11}^{E} *$ & $S_{12}^{E} *$ & $S_{13}^{E} *$ & $s_{33}^{E} *$ & $s_{44}^{E}$ & $S_{66}^{E}$ \\
\hline 8.89 & -3.15 & -2.26 & 8.97 & 24.49 & 24.08 \\
\hline$d_{31} *$ & $d_{33} *$ & $d_{15}$ & & $\varepsilon_{11}^{T}$ & $\varepsilon_{33}^{T} *$ \\
\hline-92.3 & 205 & 321.73 & & 1690 & 1824 \\
\hline \multicolumn{6}{|c|}{$(T, D)$ - type fundamental relations : $s_{i j}^{D}\left(10^{-12} \mathrm{~m}^{2} \mathrm{~N}^{-1}\right), g_{i j}\left(\mathrm{VmN}^{-1}\right), \beta_{i j}^{T}\left(10^{9} \mathrm{mF}^{-1}\right)$} \\
\hline$S_{11}^{D *}$ & $S_{12}^{D} *$ & $S_{13}^{D *}$ & $S_{33}^{D *}$ & $s_{44}^{D}$ & $s_{66}^{D}$ \\
\hline 8.35 & -3.69 & -1.09 & 6.47 & 17.72 & 24.08 \\
\hline$g_{31} *$ & $g_{33} *$ & $g_{15}$ & & $\beta_{11}^{T}$ & $\beta_{33}^{T}$ \\
\hline-0.0058 & 0.0123 & 0.021 & & 0.067 & 0.059 \\
\hline \multicolumn{4}{|c|}{ Coupling factors $k(\%)$} & \multicolumn{2}{|c|}{ Density $\left(\mathrm{kg} / \mathrm{m}^{3}\right)$} \\
\hline$k_{t} *$ & $k_{p} *$ & $k_{31} *$ & $k_{33} *$ & \multicolumn{2}{|c|}{$\rho^{*}$} \\
\hline 45.2 & 42.9 & 24.4 & 53.9 & \multicolumn{2}{|c|}{5810} \\
\hline
\end{tabular}

\section{References}

[1] [1] W.R. Eubank, F. T. Rogers, Jr., L.E. Schilberg, S. Skolnik, "Some factors influencing the dielectric properties of barium titanates" $\mathrm{J}$. $\mathrm{Am}$. Ceram. Soc., vol. 35, pp. 16-22, 1952.

[2] W. Li, Z. Xu, R. Chu, P. Fu, G. Zang, "Enhanced ferroelectric properties in $\left(\mathrm{Ba}_{1-\mathrm{x}} \mathrm{Ca}_{\mathrm{x}}\right)\left(\mathrm{Ti}_{0.94} \mathrm{Sn}_{0.06}\right) \mathrm{O}_{3}$ lead-free ceramics", J. Eur. Ceram. Soc., vol. 32(3), 517-520, 2012.

[3] T. Ikeda, "Fundamentals of Piezoelectricity", Oxford University Press, NewYork, 1996.

[4] R. Rouffaud, P. Marchet, A.-C. Hladky-Hennion, C. Bantignies, M. Pham-Thi, F. Levassort, "Complete electroelastic set for the (YXt)-45" cut of a $\mathrm{KNbO}_{3}$ single crystal”, J. Appl. Phys., vol. 116, p. 194106, 2014.

[5] H.P. Savakus, K.A. Klicker, R.E. Newnham, "PZT-epoxy piezoelectric transducers: a simplified fabrication procedure", Mater. Res. Bull., vol. 16(6), pp.677-680, 1981.
[6] ANSI/IEEE Standard on Piezoelectricity, IEEE Trans. Ultrason. Ferroelectr. Freq. Control, vol. 43, p. 717, 1996.

[7] D. Leedom, R. Krimholtz, and G. Matthaei, "New equivalent circuits for elementary piezoelectric transducers," Electronics Letters, vol. 6, no. 13, pp. 398-399, 1970.

[8] V. Yu. Topolov and C. R. Bowen, "Inconsistencies of the complete sets of electromechanical constants of relaxor-ferroelectric single crystals", J. Appl. Phys., vol. 109, p. 094107, 2011.

[9] J. H. Holland, "Adaptation in Natural and Artificial Systems", University of Michigan Press, Ann Arbor, 1975.

[10] R. Bechmann, "Elastic, Piezoelectric, and Dielectric Constants of Polarized Barium Titanate Ceramics and Some Applications of the Piezoelectric Equations", J. Acoust. Soc. Amer., vol. 28, pp. 347-350, 1956

[11] L. Zheng, X. Huo, R. Wang, j. Wang, W. Jiang and W. Cao, "Large size lead-free $(\mathrm{Na}, \mathrm{K})(\mathrm{Nb}, \mathrm{Ta}) \mathrm{O} 3$ piezoelectric single crystal : growth and full tensor properties", CrystEngComm, vol. 15, no. 38, pp. 7718-7722, 2013.

[12] F. Levassort, M. Lethiecq, D. Certon, F. Patat, "A matrix method for modeling electroelastic moduli of 0-3 piezo-composites”, IEEE Trans. Ultrason. Ferroelectr. Freq. Control, vol. 44(2), pp. 445-452, 1997. 